\title{
The Secreted Wnt Antagonist Dickkopf-1 Is Required for Amyloid $\beta$-Mediated Synaptic Loss
}

\author{
Silvia A. Purro, ${ }^{\star}$ Ellen M. Dickins, ${ }^{\star}$ and Patricia C. Salinas \\ Department of Cell and Developmental Biology, University College London, London WC1E 6BT, United Kingdom
}

Extensive evidence supports a central role for amyloid- $\beta(\mathrm{A} \beta)$ in the pathogenesis of Alzheimer's disease (AD). Synaptic loss mediated by $\mathrm{A} \beta$ in early stages of the disease might contribute to cognitive impairments. However, little is known about the mechanism by which $\mathrm{A} \beta$ induces the loss of synapses. The expression of the Wnt antagonist Dickkopf-1 (Dkk1) is increased in brains of AD patients and in AD transgenic mouse models, suggesting that dysfunction of Wnt signaling could contribute to AD pathology. Here we report that acute exposure to $\mathrm{A} \beta$ oligomers induces Dkk1 expression together with the loss of synaptic sites. Importantly, Dkk1-neutralizing antibodies suppress $\mathrm{A} \beta$-induced synapse loss in mouse brain slices. In mature rat hippocampal neurons, Dkk1 decreases the number of synapses without affecting cell viability. Ultrastructural analyses revealed that Wnt blockade decreases the size of presynaptic and postsynaptic terminals. Time-lapse recordings of RFP-labeled stable synaptic sites demonstrate that Dkk1 induces the dispersal of synaptic components. These findings identify Dkk1 as a potential therapeutic target for the treatment of AD.

\section{Introduction}

Alzheimer's disease (AD) is associated with synaptic dysfunction, the early loss of synapses and the progressive accumulation of amyloid- $\beta(\mathrm{A} \beta)$ resulting in cognitive decline (DeKosky and Scheff, 1990; Terry et al., 1991). Soluble forms of A $\beta$ decrease the number and strength of synapses before evidence of neuronal cell death (Hsia et al., 1999; Lacor et al., 2007; Shankar et al., 2007). However, the mechanisms by which $\mathrm{A} \beta$ triggers the loss of synapses are poorly understood.

A possible mechanism for $A \beta$-induced synaptic loss could involve the deregulation of factors that promote synapse formation or maintenance. Wnt secreted proteins play a key role in synaptic assembly, activity-mediated synapse remodeling and synaptic function (Gogolla et al., 2009; Inestrosa and Arenas, 2010; Budnik and Salinas, 2011). Wnt signals to both sides of the synapse to induce synaptic assembly in the CNS. In axons, Wnts activate a canonical Wnt signaling pathway that requires inhibition of Gsk3 (Ahmad-Annuar et al., 2006; Cerpa et al., 2008b; Davis et al., 2008). On dendrites, Wnts trigger the recruitment of postsynaptic components through the activation of noncanonical pathways (Farías et al., 2009; Cuitino et al., 2010; Ciani

Received Sept. 7, 2011; revised Jan. 11, 2012; accepted Jan. 24, 2012.

Author contributions: S.A.P., E.M.D., and P.C.S. designed research;S.A.P. and E.M.D. performed research; S.A.P., E.M.D., and P.C.S. analyzed data; S.A.P., E.M.D., and P.C.S. wrote the paper.

This work was supported by Alzheimer's Research UK, The Wellcome Trust, Biotechnology and Biological Sciences Research Council (PhD studentship to E.M.D.) and European Union (MOLPARK). We would like to thank Dr Matthijs Verhage for the VAMP-mRFP construct. We also thank members of our laboratory for useful discussion and comments on the manuscript.

This work is dedicated to Prof Luis E. Salinas, who died of Alzheimer's disease.

The authors declare no competing financial interests.

*S.A.P. and E.M.D. have contributed equally to the paper.

The authors declare no competing financial interests.

Correspondence should be addressed to Patricia C. Salinas, Department of Cell and Developmental Biology, University College London, London WC1E 6BT, UK. E-mail: p.salinas@ucl.ac.uk.

DOI:10.1523/JNEUROSCI.4562-11.2012

Copyright $\odot 2012$ the authors $\quad 0270-6474 / 12 / 323492-07 \$ 15.00 / 0$ et al., 2011). Given the role of Wnts in synaptic function, decreased levels of Wnt signaling could contribute to synaptic loss, characteristic of early states of AD.

Increasing evidence suggests that dysfunction of Wnt signaling is associated with AD (De Ferrari and Moon, 2006; Cerpa et al., 2008a). A variant of the Low-density lipoprotein receptor related protein 6 (LRP6), a coreceptor in the canonical Wnt pathway, has been associated with late-onset AD (De Ferrari et al., 2007). This LRP6 variant confers low levels of Wnt signaling. Consistent with reduced Wnt signaling, the secreted Wnt antagonist Dickkopf-1 (Dkk1) is elevated in postmortem $\mathrm{AD}$ brains and brains from transgenic mouse models for AD (Caricasole et al., 2004; Rosi et al., 2010). Importantly, Dkk1 blocks canonical Wnt signaling by binding to LRP6 (Niehrs, 2006). Although the mechanism of Dkk1 in AD is unclear, it has been proposed that Dkk1 might contribute to AD by promoting cell death (Caricasole et al., 2004; Rosi et al., 2010). However, given the role of Wnt signaling on synapses, elevation of Dkk1 could induce synaptic disassembly in early stages of AD.

Here we report that $D k k 1$ is required for $A \beta$-induced synapse loss. Acute exposure to $\mathrm{A} \beta$ rapidly increases Dkk1 mRNA levels in hippocampal brain slices, at the time when synapses are lost. Neutralizing antibodies against Dkk1 block synaptic loss induced by $\mathrm{A} \beta$. In mature hippocampal neurons, Dkk1 rapidly induces the loss of synapses without affecting cell viability. Ultrastructural analyses reveal that the remaining synapses are smaller after Wnt blockade with Dkk1. Time-lapse microscopy of RFP-labeled synaptic sites reveals that Dkk1 induces synaptic disassembly through the dispersal of synaptic components. These results indicate that Wnt signaling is involved in synaptic maintenance. Moreover, our studies demonstrate that Dkk1 mediates $\mathrm{A} \beta$-induced synapse disassembly and suggest that blockade of Dkk1 could be beneficial for the maintenance of synapses in Alzheimer's disease. 


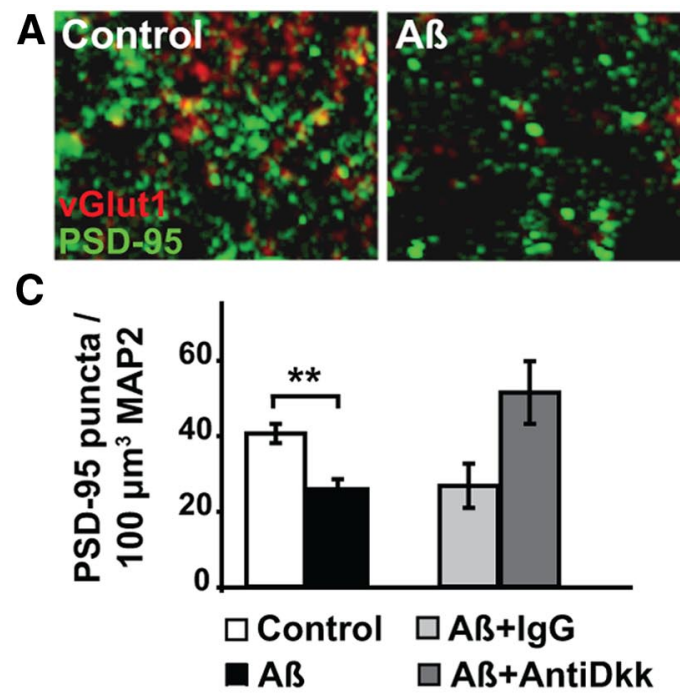

$\mathbf{E}$

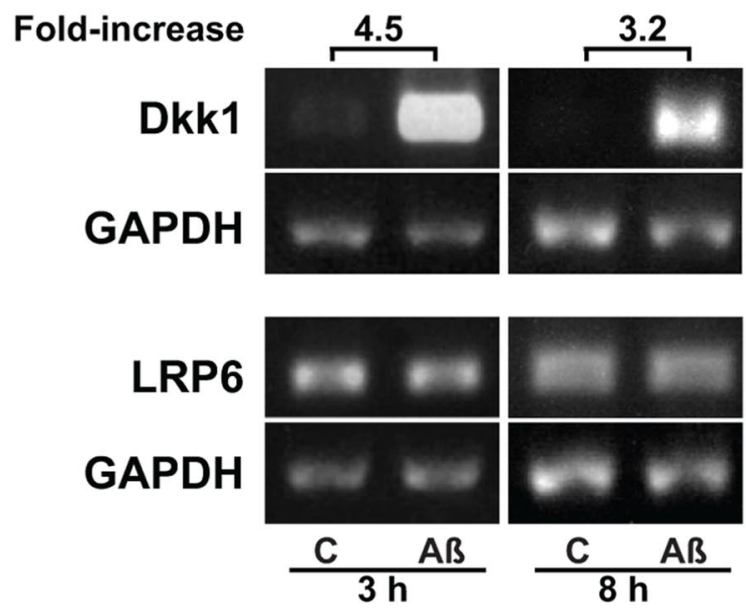

$\mathbf{B}$
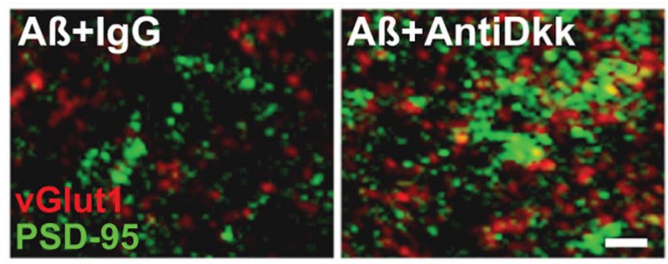

D
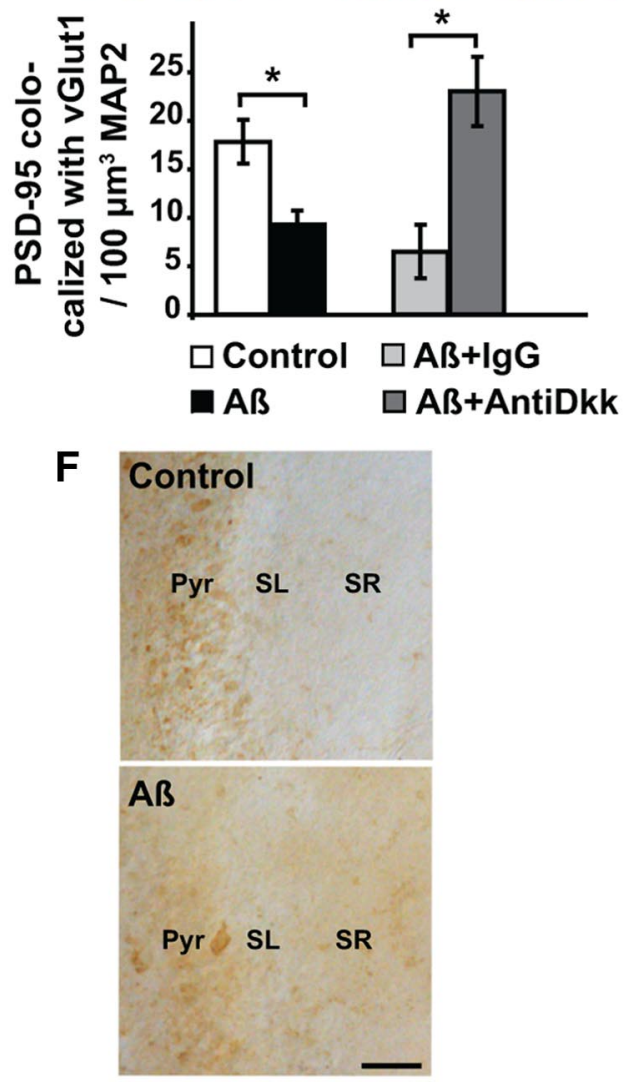

Figure 1. Dkk1 is required for synaptic loss induced by $A \beta . A, B$, Acute hippocampal slices from adult mice were treated with vehicle, $5 \mu \mathrm{m} A \beta(A), A \beta+$ control lgG, or $A \beta+$ anti-Dkk1 antibody $(B)$ for $3 \mathrm{~h}$. Scale bar, $5 \mu \mathrm{m}$. C, D, Quantifications show that blockade of Dkk1 abolishes synaptic loss by A $\beta$. $E$, RT-PCR analyses show that A $\beta$ significantly increases the level of Dkk1 expression at 3 and $8 \mathrm{~h}$ without affecting LRP6 levels ( $n=5$ mice; a representative sample is shown here). $\boldsymbol{F}$, Dkk1 protein level increases after exposure to $A \beta$ for $3 \mathrm{~h}$ in stratum lucidum (SL) and stratum radiatum (SR) of the CA3 region. Pyr, Pyramidal layer. Scale bar, $60 \mu \mathrm{m} .{ }^{*} p<0.05,{ }^{* *} p<0.005$. Error bars represent SEM.

\section{Materials and Methods}

Neuronal cultures. Rat hippocampal cultures were prepared as described previously (Dotti et al., 1988). Hippocampal dissociated neurons were cultured in Neurobasal medium supplemented with N2/B27. Neurons were exposed to $20 \mathrm{ng} / \mathrm{ml} \mathrm{Dkk1}$ (R\&D Systems) for $2 \mathrm{~h}$. Apoptosis was determined by TUNEL (Apo-Tag; Millipore Bioscience Research Reagents), DNase-I treatment (Roche) was used as positive control. For time-lapse experiments, hippocampal cells were electroporated (Amaxa Biosystems) with VAMPmRFP construct. After 21-28 d in culture, time-lapse recordings were performed. For immunofluorescence, the following antibodies were used: VAMP2 (Synaptic Systems), Bassoon (Bioquote Limited), PSD-95 and GRIP (Thermo Scientific), vGlut-1, $\beta$-III tubulin (Tuj-1) and Cask (Millipore Bioscience Research Reagents). Alexa Fluor 488-, Alexa Fluor 546-, and Alexa Fluor 647-conjugated secondary antibodies were from Invitrogen. FM1-43 (Invitrogen) uptake was performed as described previously (Ahmad-Annuar et al., 2006).

Brain slices and $A \beta$ oligomers ( $A \beta$-derived diffusible ligands). ADDLs $(\mathrm{A} \beta$-derived diffusible ligands) were prepared as previously described (Klein, 2002). Sagittal hippocampal slices $(300 \mu \mathrm{m})$ from 6- to 13month-old mice of either sex were cut in ice-cold aCSF with a vibratome (Campden Instruments). After $1 \mathrm{~h}$ recovery at $37^{\circ} \mathrm{C}$, slices were incubated in aCSF with vehicle (Neurobasal phenol red-free medium) or 5 $\mu \mathrm{M}$ ADDLs for 3 or $8 \mathrm{~h}$. Slices were either fixed with 4\% PFA or resuspended in TRIzol (Invitrogen) for reverse transcriptase (RT)-PCR assays. Anti-Dkk1 (R\&D Systems) or control goat IgG (Santa Cruz Biotechnology) were used at $1 \mu \mathrm{g} / \mathrm{ml}$. For localization of Dkk1 protein, anti-Dkk1 and anti-goat peroxidase-conjugated antibodies Vectastain $\mathrm{ABC}$ kit (Vector Labs) was used according manufacture's protocol.

Time-lapse recordings. Time-lapse experiments were performed on a Leica TCS SP1 confocal microscope with a heated stage and $\mathrm{CO}_{2}$ chamber. Eight to 10 frame stacks were acquired at $1 \mathrm{~min}$ interval. Stable VAMP2-mRFP puncta were identified as those that did not change in size or move for a period of $20 \mathrm{~min}$ before addition of Dkk1. Data from 2 independent experiments under control conditions (28 stable puncta) and 3 independent experiments with Dkk1 treatment (53 stable puncta) were analyzed. For kymographs, a line of 15 pixels wide was traced along the selected process.

Image analysis and quantification. Confocal images were acquired and analyzed using MetaMorph or Volocity software. For acute brain slices, confocal images from the CA3 apical region were analyzed from 20 to 30 brain slices per condition (from 5 independent experiments). For dissociated cultures, 10-15 random fields were acquired and analyzed per condition (from at least 3 independent experiments). A threshold was set to capture clusters that were clearly distinguishable 
and did not merge with one another. Synaptic puncta were normalized either by neurite length or to area of tubulin in case of $2 \mathrm{D}$ images depending of cell density, and to MAP2 or tubulin volume in the case of confocal 3D images. The number, size, and colocalization of puncta were measured and analyzed by two-way ANOVA.

Electron microscopy. Hippocampal neurons (21 d in vitro) were treated with Dkk1 or vehicle for $2 \mathrm{~h}$ and then prepared for electron microscopy according to a previously described protocol (Svitkina et al., 1995). Electron micrographs were acquired in a JEOL1010 electron microscope at a magnification of $60,000 \times$. PSD area was traced manually for quantifications.

\section{Results}

$A \beta$ oligomers rapidly induce synaptic loss and expression of Dkk1 in acute hippocampal slices

Here we investigated the contribution of decreased Wnt signaling to $A \beta$-mediated synaptic loss. The expression of the secreted Wnt antagonist Dkk1 is increased in the hippocampus of transgenic mouse models for $\mathrm{AD}$ (Rosi et al., 2010). We therefore examined whether $\mathrm{A} \beta$ directly regulates Dkk 1 expression in acute brain slices by using $\mathrm{A} \beta$ derived diffusible ligands (defined here as $A \beta$ ). Short-term exposure ( $3 \mathrm{~h}$ ) to $A \beta$ rapidly induces loss of synapses indicated by a decrease in the number of the postsynaptic marker PSD95 and the colocalization of vGlut1 (presynaptic marker) and PSD-95 puncta (Fig. $1 A ; C, D$ ). RT-PCR analyses also revealed that $\mathrm{A} \beta$ increases the level of Dkk1 mRNA in a sustained manner, without affecting LRP6 receptor levels (Fig. 1E). Moreover, Dkk1 protein levels are also increased in the CA3 (Fig. 1F), DG and CA1 areas (data not shown). These results suggest that $\mathrm{A} \beta$ could affect synapses by blocking Wnt signaling through Dkk1.

To directly test the contribution of Dkk1 on $\mathrm{A} \beta$-mediated synaptic loss, we used a neutralizing antibody against Dkk1 (Yaccoby et al., 2007). Although control IgGs do not affect the reduction in synapse density by $\mathrm{A} \beta$, specific anti-Dkk1 antibodies completely abolish the effect of $A \beta$ on synapses (Fig. $1 B ; C, D$ ). Indeed, $\mathrm{A} \beta$ decreases the percentage of synapses and this effect is suppressed by blocking Dkk1 (Control, $46.2 \pm$ 5.4\%; $\mathrm{A} \beta+\operatorname{IgG}, 19.4 \pm 4.9 \% ; \mathrm{A} \beta+$ AntiDkk1, $44.3 \pm 4.6 \%, p<0.05)$. Under basal conditions, blockade of Dkk1 does no affect the number of synapses (data not shown) consistent with the finding that Dkk1 is expressed at very low levels in the hippocampus. Moreover, no changes in the level of cell death were observed in acute brain slices under any of the experimental conditions. Together, these findings demonstrate that Dkk1 mediates the synaptic loss induced by short-term exposure to $\mathrm{A} \beta$.

B
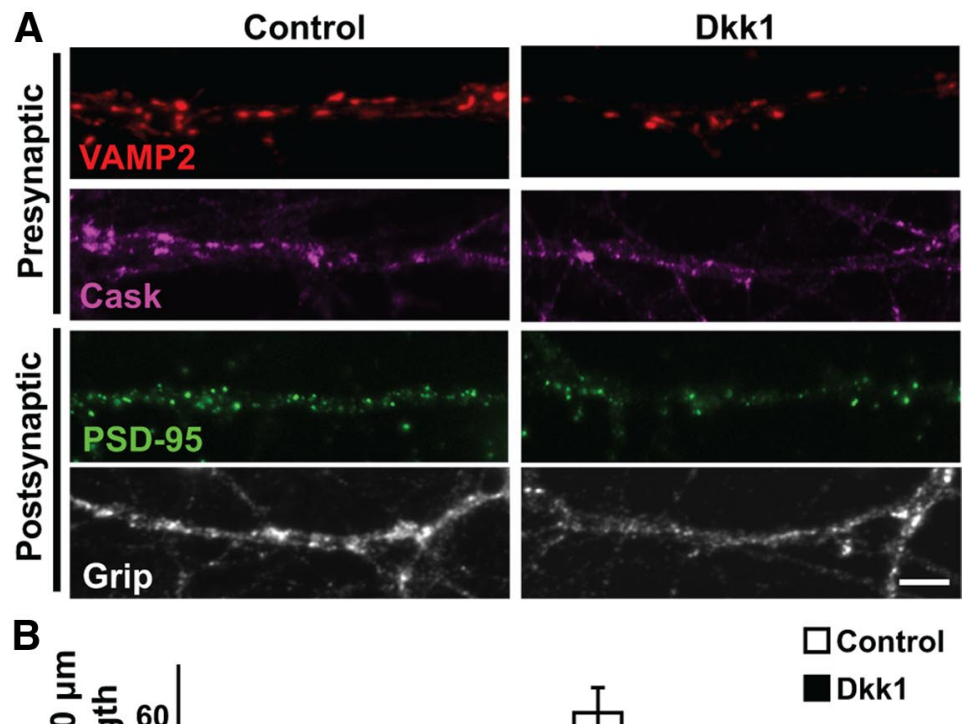

$\square$ Control
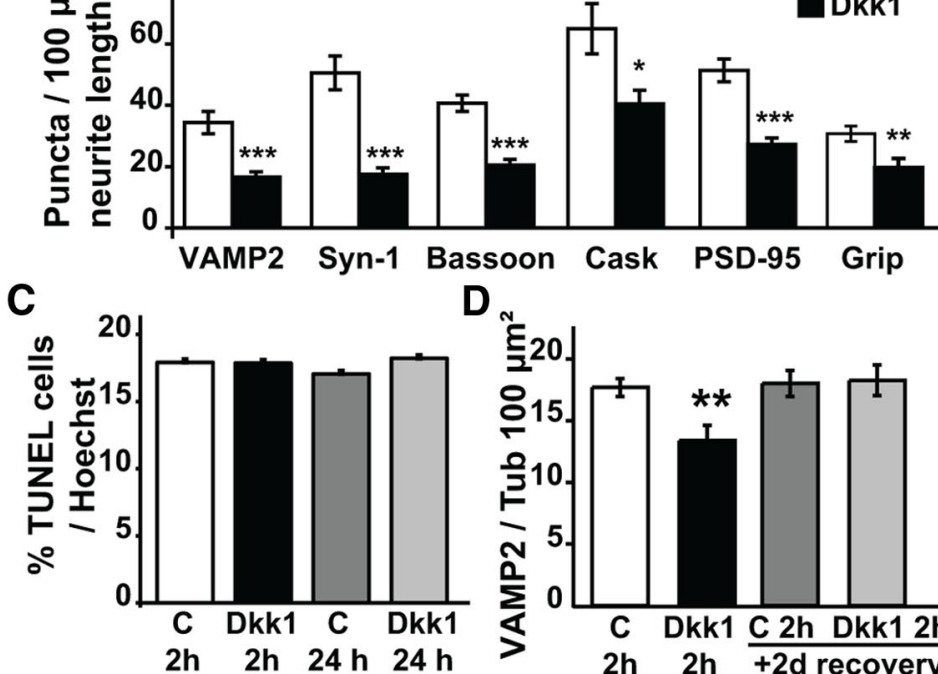

$D^{\circ}$
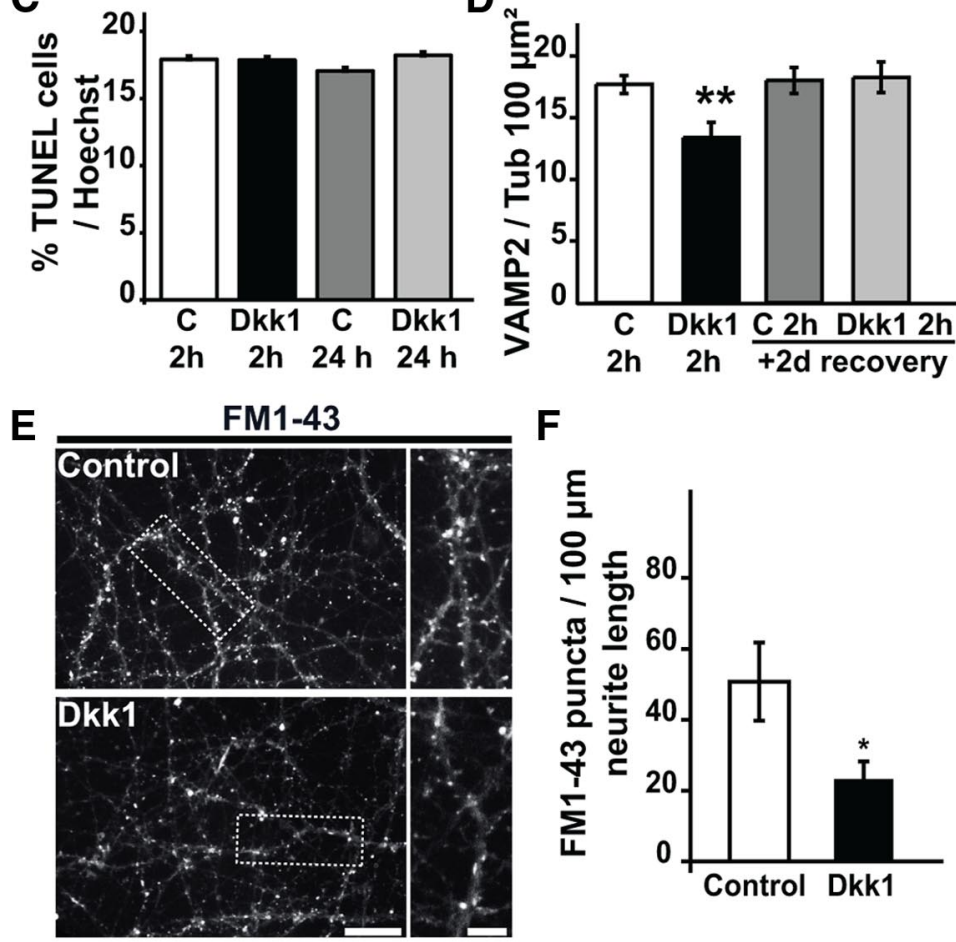

$F$

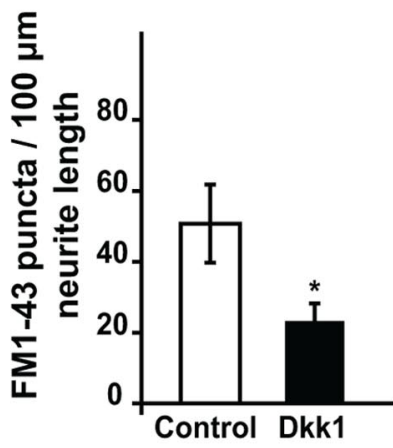

Figure 2. Dkk1 rapidly and reversibly reduces the number of synaptic sites in hippocampal neurons. $\boldsymbol{A}, \boldsymbol{B}$, Immunofluorescence microscopy $(\boldsymbol{A})$ and quantification $(\boldsymbol{B})$ of the number of several presynaptic and postsynaptic protein clusters in hippocampal neurons treated with vehicle or Dkk1 for $2 \mathrm{~h}$. Scale bar, $5 \mu \mathrm{m}$. Syn-1, Synapsin-1. C, Hippocampal cells were treated with Dkk1 for 2 or $24 \mathrm{~h}$, then TUNEL assays were performed, counterstained with Hoechst. C, Control. D, Hippocampal cells were treated with Dkk1 or control media for $2 \mathrm{~h}$, then Dkk1 was removed and the cells incubated for another $2 \mathrm{~d}$ in the absence of Dkk1. Quantification shows that the effect of Dkk1 is reversible. C, Control. $\boldsymbol{E}, \boldsymbol{F}, \mathrm{FM} 1-43$ uptake shows that Dkk1 decreases the number of synaptic vesicle recycling sites (puncta). Scale bars: left, $20 \mu \mathrm{m}$; right, $10 \mu \mathrm{m} .{ }^{*} p<0.05,{ }^{* *} p<0.005,{ }^{* * *} p<0.001$. Error bars represent SEM.
Inhibition of Wnt signaling reduces the number of functional synaptic sites

We next examined the effect of Dkk1 on synapses. In hippocampal dissociated neurons, Dkk1 decreases the number of presynaptic 

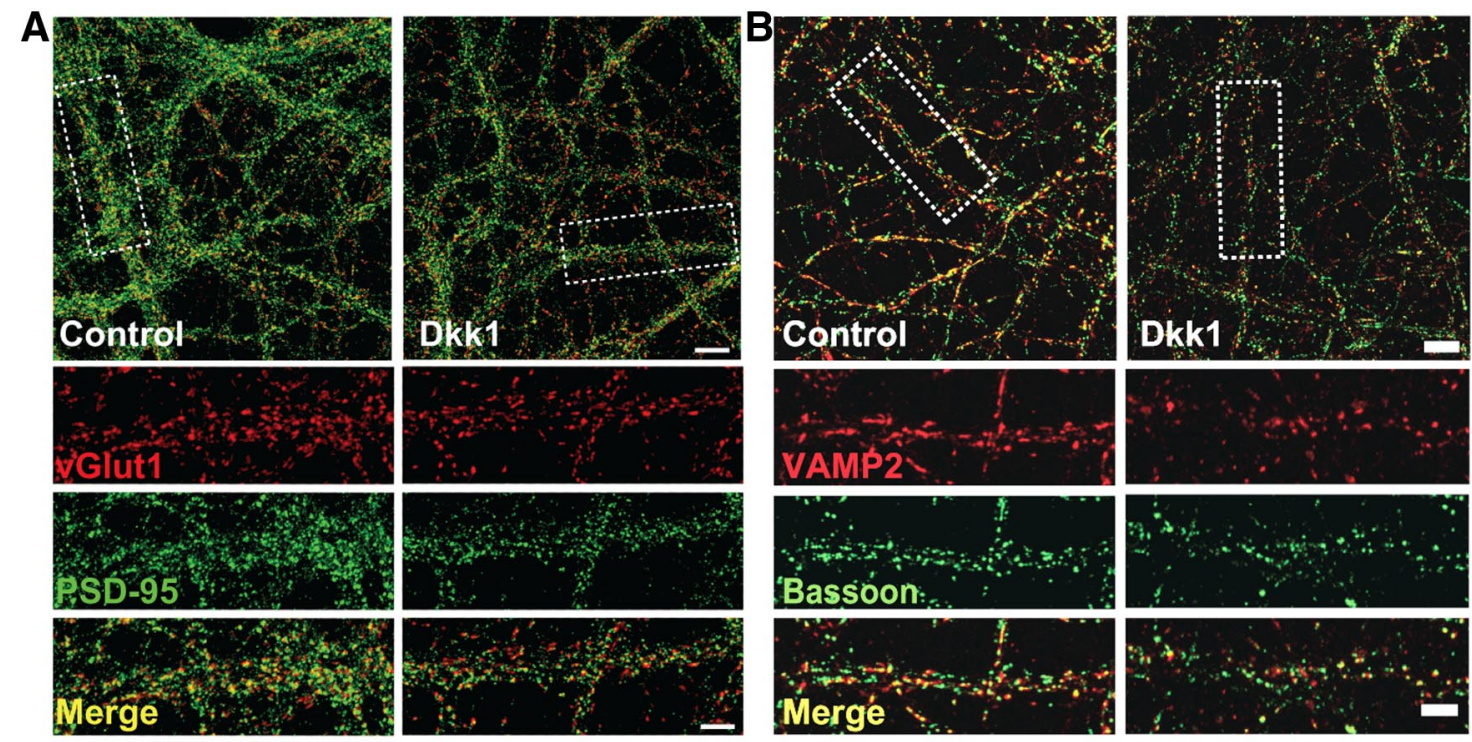

C
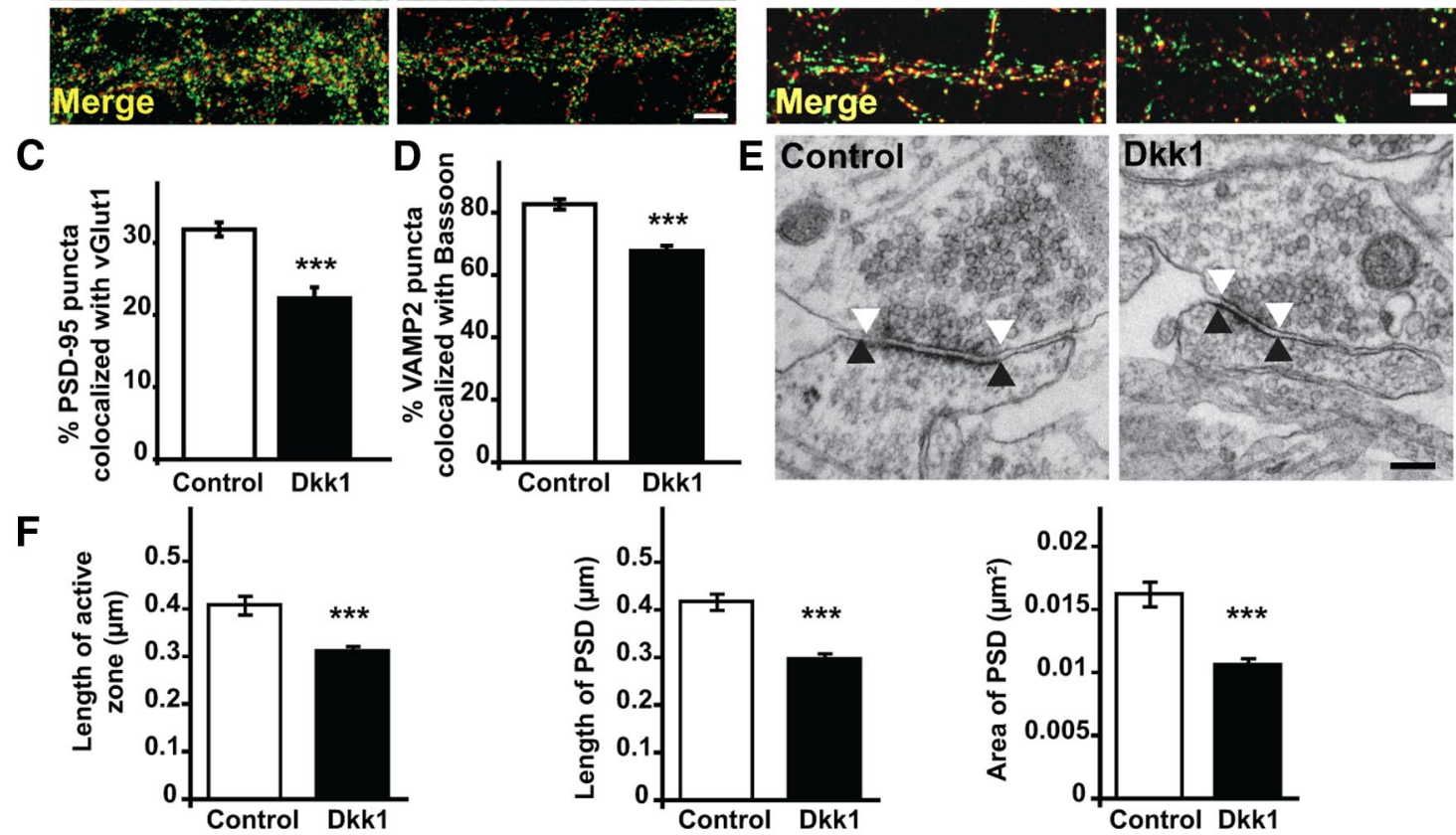

Figure 3. Dkk1 induces disassembly of synaptic sites. Mature hippocampal neurons were treated with Dkk1. Images $(A)$ and quantification $(C)$ show that Dkk1 reduces the colocalization of the postsynaptic marker PSD-95 with the presynaptic marker vGlut1. Scale bars: top, $10 \mu \mathrm{m}$; bottom, $5 \mu \mathrm{m}$. Images $(\boldsymbol{B})$ and quantification $(\boldsymbol{D})$ reveal that Wnt blockade decreases the colocalization of two presynaptic markers, VAMP2 and Bassoon. Scale bars: top, $10 \mu \mathrm{m}$; bottom, $5 \mu \mathrm{m}$. $\boldsymbol{E}$, Electron micrographs of vehicle- and Dkk1-treated neurons. White and black arrowheads, Length of the active zone (AZ) and the postsynaptic density region (PSD), respectively. Scale bar, $0.2 \mu \mathrm{m} . \boldsymbol{F}$, Quantification reveals that Dkk1 significantly reduces the AZ length, PSD length, and PSD area. ${ }^{* * *} p<$ 0.001. Error bars represent SEM.

markers such as VAMP2, Synapsin-1, Bassoon, and Cask by $52,65,50$, and $38 \%$, respectively, as well as the number of postsynaptic markers PSD-95 and Grip (47\% and 34\%, respectively) (Fig. $2 A, B$ ). This effect was not due to changes in cell viability, as Dkk1 does not affect the number of TUNELpositive neurons (Fig. 2C). Importantly, removal of Dkk1 results in the recovery of synaptic puncta number to control levels (Fig. 2D). Thus, Dkk1 rapidly but reversibly decreases the number of presynaptic and postsynaptic sites.

We used the uptake of the styryl dye, FM1-43 to measure the effect of Dkk1 on the number of release sites. Hippocampal neurons treated with control or Dkk1 were stimulated with $\mathrm{KCl}$ in the presence of FM1-43. In the presence of Dkk1, neurons exhibit a significant reduction $(53 \%)$ in the number of FM1-43-positive puncta (Fig. 2E,F) when compared with controls, indicating that Dkk1 reduces the number of functional presynaptic sites.
Wnt blockade induces synaptic disassembly in mature neurons

We next examined whether changes in synaptic protein levels could account for the changes in the number of synaptic puncta induced by Dkk1. Western blot analyses showed that Dkk1 does not affect the levels of synaptic proteins including Synapsin-1, VAMP2, Cask or PSD-95 (data not shown). These data suggest that Dkk1 decreases the number of synaptic sites by inducing the disassembly of synapses rather than protein degradation. To test this hypothesis, we examined the colocalization of presynaptic and postsynaptic markers (Fig. $3 A$ ). Indeed, Dkk1 decreases by $30 \%$ the number of colocalized vGlut 1 and PSD-95 puncta (Fig. 3C), indicating a decrease in the apposition of presynaptic and postsynaptic components.

If Dkk1 induces synaptic disassembly, synaptic components present on the same presynaptic site should also delocalize. In mature neurons, the vast majority of puncta for the 

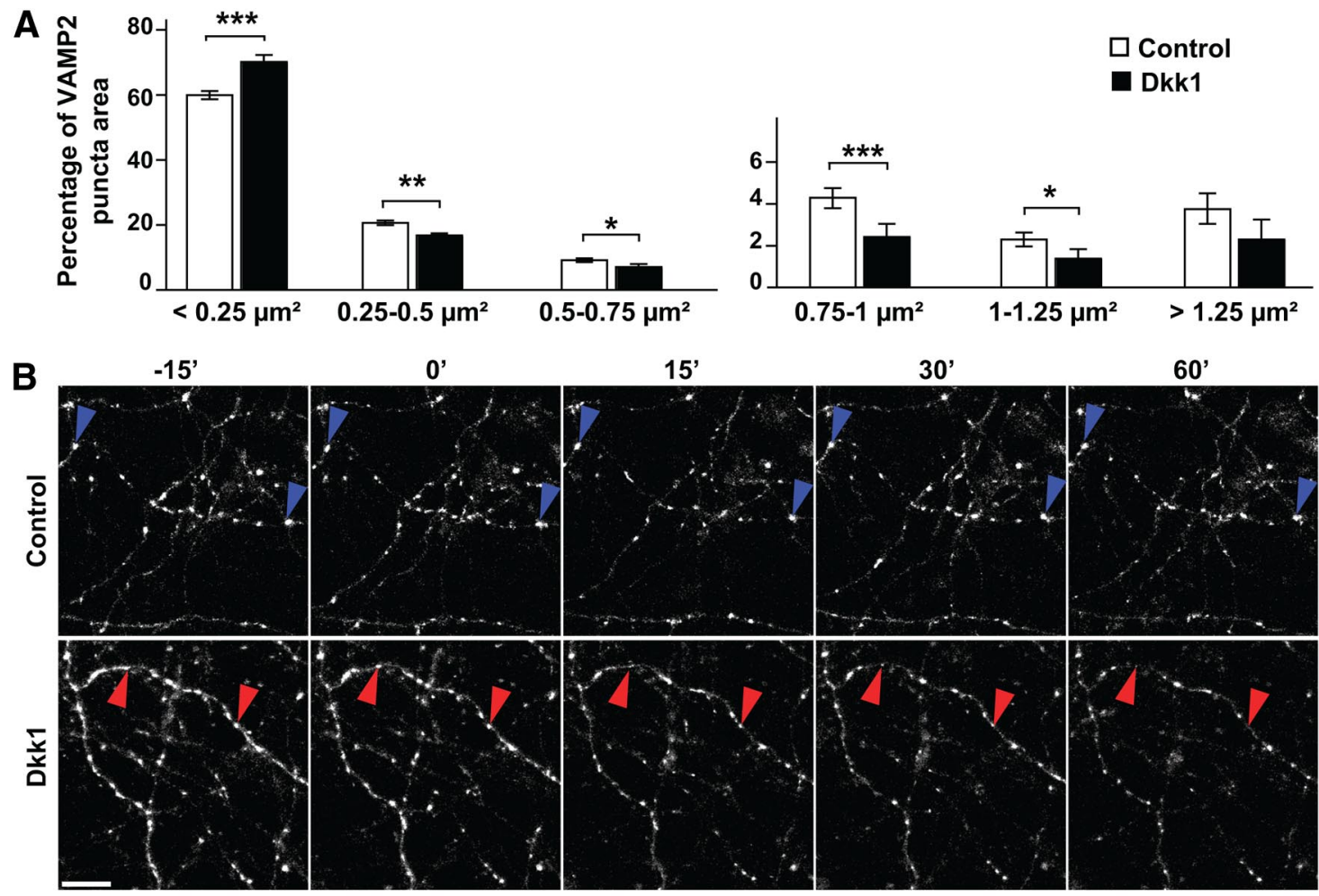

C
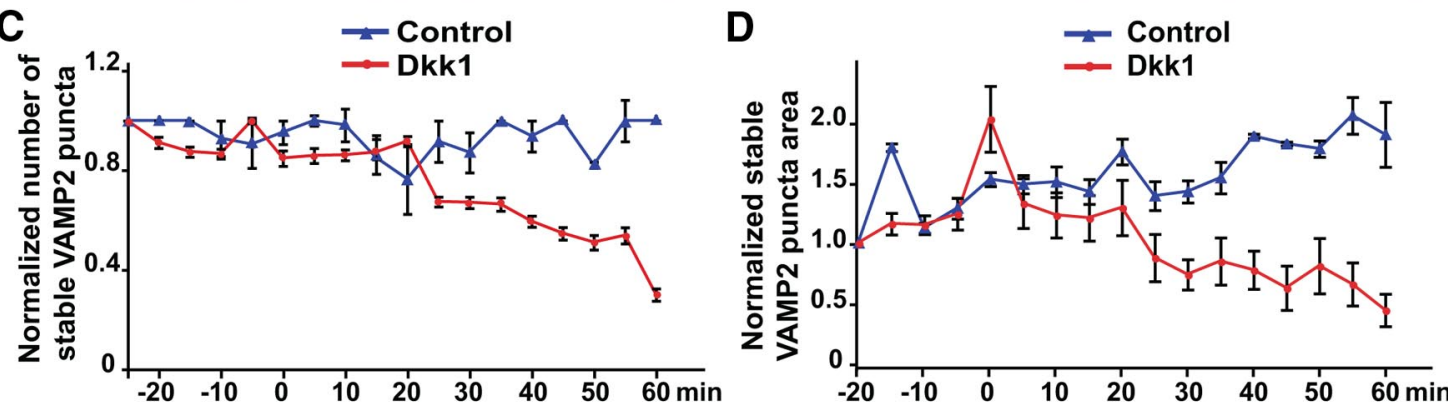

$\mathbf{E}$
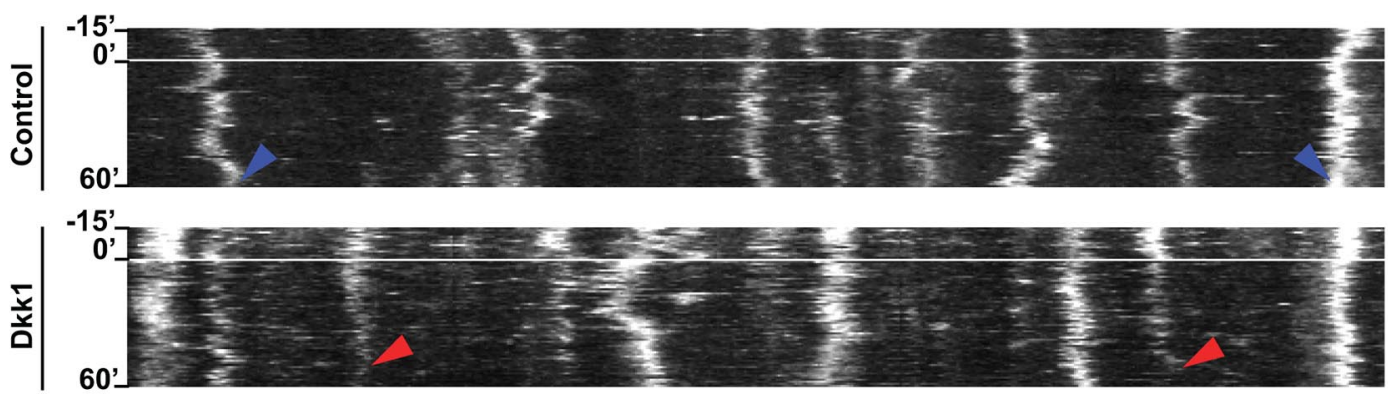

Figure 4. Dkk1 induces the dispersal of synaptic components. $A$, Size distribution of VAMP2 puncta after short-term treatment with Dkk1. B, Mature hippocampal cells expressing VAMP2-mRFP were recorded for $20 \mathrm{~min}$ and then Dkk1 or control media was added for $1 \mathrm{~h}$. Scale bar, $10 \mu \mathrm{m}$. C, D, Quantifications show the effect of Dkk 1 in the number and size of stable VAMP2-mFRP clusters. $E$, Kymographs along a single process from control or Dkk1-treated neurons. The white horizontal line indicates when Dkk1 or control media was added. Blue and red arrowheads indicate stable or lost (previously stable) puncta, respectively. ${ }^{*} p<0.05,{ }^{* *} p<0.005,{ }^{* * *} p<0.0005$. Error bars represent SEM.

synaptic vesicle protein VAMP2 colocalize with the active zone protein Bassoon (Fig. $3 B$ ). In contrast, in the presence of Dkk1, the level of colocalization of these two markers decreases by $20 \%$ (Fig. 3D). Together, our findings indicate that Dkk1 induces the dispersal of synaptic proteins at both sides of the synapse in mature neurons.

\section{Dkk1 reduces the size of synapses in mature neurons}

The robust and rapid decrease in the number of synaptic sites induced by Dkk1 suggests that this Wnt antagonist not only blocks endogenous synaptogenic activity, but also affects existing synapses. We therefore examined the effect of Dkk1 on synapses at the ultrastructural level. Only structures that fulfilled the classical criteria of an asymmetric synapse (i.e., the presence of a homogeneous pool of synaptic vesicles associated with plasma membrane in direct apposition to a defined postsynaptic density (PSD) and separated by a defined synaptic cleft) were analyzed by electron microscopy (Harris and Rosenberg, 1993). Therefore, synapses in advanced stages of disassembly were excluded. EM analyses revealed that Dkk1 
induces a significant reduction in the length of the active zone $(23 \%)$ and in the length $(29 \%)$ and area (35\%) of the PSD (Fig. $3 E, F)$. Thus, Dkk1 induces a considerable decrease in the size of synapses in mature dissociated neurons.

\section{Time-lapse recordings revealed that Dkk1 rapidly induces synaptic disassembly}

To further characterize the effect of Dkk1 in synaptic disassembly, we measured the size of synaptic vesicle clusters. If Dkk1 promotes dispersal of synaptic components, an increase in the number of small clusters with the concomitant decrease in larger ones should be observed. Indeed, Dkk1 induces a $17 \%$ increase in the number of small puncta with a concomitant $40 \%$ decrease in the number of larger clusters (Fig. 4A). These results together with the EM studies strongly suggest that blockade of Wnt signaling results in the shrinkage of synapses.

We next examined the dynamics of Dkk1-induced synaptic disassembly by time-lapse recordings of hippocampal dissociated neurons expressing VAMP2-mRFP to label presynaptic sites. In mature hippocampal neurons, the majority of VAMP2-mRFP puncta corresponds to synaptic sites as they colocalize with PSD-95 puncta (data not shown). Stable puncta were identified by imaging for $20 \mathrm{~min}$ before addition of Dkk1 or control media. VAMP2-mRFP puncta that remained at the same position along the neurite were classified as "stable" and were selected for analysis. In contrast, moving puncta were classified as "in transit" and not included in our analyses. Kymographs showed that under basal conditions VAMP2-mRFP stable puncta displayed the same intensity during the recorded period indicating that there was not significant photobleaching during the recording (Fig. $4 E$ ). As expected for mature neurons, the number of VAMP2-mRFP stable puncta does not change significantly during the $60 \mathrm{~min}$ recording in control neurons (Fig. $4 B, C$ ). In the presence of Dkk1, however, $70 \%$ of stable puncta are lost (Fig. $4 B, C$ ). The effect of Dkk1 was rapid as the number of clusters decreases just after the first 25 min of treatment (Fig. 4C). Analyses of the puncta population in control neurons revealed that although many stable puncta fluctuated in size, overall they exhibited a consistent increase in size over $1 \mathrm{~h}$ recording (Fig. $4 D$ ). In contrast, Dkk1 induces a $78 \%$ reduction in the average area of stable clusters (Fig. $4 D$ ). Analyses of the initial and final size of individual puncta revealed that most small puncta increase in size under control conditions whereas large puncta largely remained unchanged. In contrast, Dkk1 reduces the size of small and large puncta (data not shown). Together, these findings demonstrate that Dkk1 induces a rapid synaptic disassembly by inducing the dispersal of synaptic components.

\section{Discussion}

Loss of synapses is an early event in $\mathrm{AD}$, a process that might contribute to the cognitive decline characteristic of this disease (Selkoe, 2002). A $\beta$ is considered the primary factor that induces synaptic loss and dysfunction. Natural oligomers or ADDLs induce the loss of synapses in brain slices and in dissociated hippocampal cultures (Lacor et al., 2007; Shankar et al., 2007). Moreover, decreased levels of synaptic markers and dendritic spines have been observed in transgenic AD mouse models (Knobloch and Mansuy, 2008). Decreased synapse density and deficits in synaptic plasticity are evident before neuronal degeneration is apparent (Hardy and Selkoe, 2002; Klein, 2002; Walsh and Selkoe, 2004). However, the mechanisms by which $A \beta$ induces synaptic loss are not fully understood. Here we present evidence that Dkk1, which is elevated by A $\beta$, induces synapse disassembly. Im- portantly, blockade of Dkk1 protects against $\mathrm{A} \beta$-mediated synaptic loss.

The potential link between Wnt signaling and $\mathrm{AD}$ has been well documented. Several studies have demonstrated that Wnt ligands can protect against $\mathrm{A} \beta$-induced neuronal degeneration in cultured neurons (De Ferrari et al., 2003; Cerpa et al., 2010). In addition, dysfunction of canonical Wnt signaling, which signals through LRP6 to inhibit Gsk3, might contribute to AD pathology by inducing Tau hyperphosphorylation (Hooper et al., 2008) or by affecting APP (A $\beta$ precursor protein) processing (Mudher et al., 2001). As mentioned before, a genetic variant of LRP6 conferring low levels of Wnt signaling is correlated with late-onset AD (De Ferrari et al., 2007). Moreover, the levels of the canonical Wnt antagonist Dkk1 are increased in brains of AD patients and in transgenic model of $\mathrm{AD}$ (Caricasole et al., 2004; Rosi et al., 2010 ). Here we demonstrate that $A \beta$ rapidly increases the levels of Dkk1 in the hippocampus, which is required for $A \beta$ to induce synapse disassembly, as blockade of Dkk1 suppresses this effect.

How does Dkk1 induce disassembly? This Wnt antagonist rapidly but reversibly induces synaptic disassembly at both sides of the synapse. In our system, Dkk1 reversibly affects the number of synapses without affecting programmed cell death. Interestingly, previous studies have indicated that increased Dkk1 levels contribute to cell death in cerebral ischemia (Cappuccio et al., 2005; Mastroiacovo et al., 2009), epilepsy (Busceti et al., 2007) and neurodegenerative diseases (Scali et al., 2006; Rosi et al., 2010). This apparent discrepancy could be explained by the low level and short-term treatment with Dkk1 used in our system. Given the rapid increase of Dkk1 expression by $\mathrm{A} \beta$ and that cell death is a late event in $\mathrm{AD}$ resulting from synaptic dysfunction, our studies suggest that Dkk1 modulates synaptic loss without cell death in the early stages of AD.

Dkk1 triggers synapse loss by inducing the dispersal of synaptic components. In mature neurons, a large proportion of synapses disassemble in the presence of Dkk1 but those that remain are smaller at the ultrastructural level. Time-lapse recordings of VAMP-mRFP labeled synaptic puncta demonstrate that Dkk1 rapidly decreases the number, size and intensity of stable synaptic sites. The speed of this event is similar to those reported during activity-induced synaptic disassembly (Hopf et al., 2002). As Dkk1 functions by blocking Wnt signaling through binding to the LRP6 receptor, these studies demonstrate a role for canonical Wnt signaling in the maintenance of central synapses. In summary, we demonstrate that Dkk1 is a critical participant in synaptic disassembly induced by $\mathrm{A} \beta$ and propose that suppression of Dkk1 or increasing Wnt signaling could be beneficial for the treatment of Alzheimer's disease.

\section{References}

Ahmad-Annuar A, Ciani L, Simeonidis I, Herreros J, Fredj NB, Rosso SB, Hall A, Brickley S, Salinas PC (2006) Signaling across the synapse: a role for Wnt and Dishevelled in presynaptic assembly and neurotransmitter release. J Cell Biol 174:127-139.

Budnik V, Salinas PC (2011) Wnt signaling during synaptic development and plasticity. Curr Opin Neurobiol 21:151-159.

Busceti CL, Biagioni F, Aronica E, Riozzi B, Storto M, Battaglia G, Giorgi FS, Gradini R, Fornai F, Caricasole A, Nicoletti F, Bruno V (2007) Induction of the Wnt inhibitor, Dickkopf-1, is associated with neurodegeneration related to temporal lobe epilepsy. Epilepsia 48:694-705.

Cappuccio I, Calderone A, Busceti CL, Biagioni F, Pontarelli F, Bruno V, Storto M, Terstappen GT, Gaviraghi G, Fornai F, Battaglia G, Melchiorri D, Zukin S, Nicoletti F, Caricasole A (2005) Induction of Dickkopf-1, a negative modulator of the Wnt pathway, is required for the development of ischemic neuronal death. J Neurosci 25:2647-2657.

Caricasole A, Copani A, Caraci F, Aronica E, Rozemuller AJ, Caruso A, Storto 
M, Gaviraghi G, Terstappen GC, Nicoletti F (2004) Induction of Dickkopf-1, a negative modulator of the Wnt pathway, is associated with neuronal degeneration in Alzheimer's brain. J Neurosci 24:6021-6027.

Cerpa W, Dinamarca MC, Inestrosa NC (2008a) Structure-function implications in Alzheimer's disease: effect of Abeta oligomers at central synapses. Curr Alzheimer Res 5:233-243.

Cerpa W, Godoy JA, Alfaro I, Farías GG, Metcalfe MJ, Fuentealba R, Bonansco C, Inestrosa NC (2008b) Wnt-7a modulates the synaptic vesicle cycle and synaptic transmission in hippocampal neurons. J Biol Chem 283:5918-5927.

Cerpa W, Farías GG, Godoy JA, Fuenzalida M, Bonansco C, Inestrosa NC (2010) Wnt-5a occludes Abeta oligomer-induced depression of glutamatergic transmission in hippocampal neurons. Mol Neurodegener 5:3.

Ciani L, Boyle KA, Dickins E, Sahores M, Anane D, Lopes DM, Gibb AJ, Salinas PC (2011) Wnt7a signaling promotes dendritic spine growth and synaptic strength through $\mathrm{Ca}^{2+} /$ Calmodulin-dependent protein kinase II. Proc Natl Acad Sci U S A 108:10732-10737.

Cuitino L, Godoy JA, Farías GG, Couve A, Bonansco C, Fuenzalida M, Inestrosa NC (2010) Wnt-5a modulates recycling of functional GABAA receptors on hippocampal neurons. J Neurosci 30:8411-8420.

Davis EK, Zou Y, Ghosh A (2008) Wnts acting through canonical and noncanonical signaling pathways exert opposite effects on hippocampal synapse formation. Neural Dev 3:32.

De Ferrari GV, Moon RT (2006) The ups and downs of Wnt signaling in prevalent neurological disorders. Oncogene 25:7545-7553.

De Ferrari GV, Chacón MA, Barría MI, Garrido JL, Godoy JA, Olivares G, Reyes AE, Alvarez A, Bronfman M, Inestrosa NC (2003) Activation of Wnt signaling rescues neurodegeneration and behavioral impairments induced by beta-amyloid fibrils. Mol Psychiatry 8:195-208.

De Ferrari GV, Papassotiropoulos A, Biechele T, Wavrant De-Vrieze F, Avila ME, Major MB, Myers A, Sáez K, Henríquez JP, Zhao A, Wollmer MA, Nitsch RM, Hock C, Morris CM, Hardy J, Moon RT (2007) Common genetic variation within the low-density lipoprotein receptor-related protein 6 and late-onset Alzheimer's disease. Proc Natl Acad Sci U S A 104:9434-9439.

DeKosky ST, Scheff SW (1990) Synapse loss in frontal cortex biopsies in Alzheimer's disease: correlation with cognitive severity. Ann Neurol $27: 457-464$.

Dotti CG, Sullivan CA, Banker GA (1988) The establishment of polarity by hippocampal neurons in culture. J Neurosci 8:1454-1468.

Farías GG, Alfaro IE, Cerpa W, Grabowski CP, Godoy JA, Bonansco C, Inestrosa NC (2009) Wnt-5a/JNK signaling promotes the clustering of PSD-95 in hippocampal neurons. J Biol Chem 284:15857-15866.

Gogolla N, Galimberti I, Deguchi Y, Caroni P (2009) Wnt signaling mediates experience-related regulation of synapse numbers and mossy fiber connectivities in the adult hippocampus. Neuron 62:510-525.

Hardy J, Selkoe DJ (2002) The amyloid hypothesis of Alzheimer's disease: progress and problems on the road to therapeutics. Science 297:353-356.

Harris KM, Rosenberg PA (1993) Localization of synapses in rat cortical cultures. Neuroscience 53:495-508.

Hooper C, Killick R, Lovestone S (2008) The GSK3 hypothesis of Alzheimer's disease. J Neurochem 104:1433-1439.

Hopf FW, Waters J, Mehta S, Smith SJ (2002) Stability and plasticity of developing synapses in hippocampal neuronal cultures. J Neurosci 22:775-781.

Hsia AY, Masliah E, McConlogue L, Yu GQ, Tatsuno G, Hu K, Kholodenko D, Malenka RC, Nicoll RA, Mucke L (1999) Plaque-independent disruption of neural circuits in Alzheimer's disease mouse models. Proc Natl Acad Sci U S A 96:3228-3233.

Inestrosa NC, Arenas E (2010) Emerging roles of Wnts in the adult nervous system. Nat Rev Neurosci 11:77-86.

Klein WL (2002) Abeta toxicity in Alzheimer's disease: globular oligomers (ADDLs) as new vaccine and drug targets. Neurochem Int 41:345-352.

Knobloch M, Mansuy IM (2008) Dendritic spine loss and synaptic alterations in Alzheimer's disease. Mol Neurobiol 37:73-82.

Lacor PN, Buniel MC, Furlow PW, Clemente AS, Velasco PT, Wood M, Viola KL, Klein WL (2007) Abeta oligomer-induced aberrations in synapse composition, shape, and density provide a molecular basis for loss of connectivity in Alzheimer's disease. J Neurosci 27:796-807.

Mastroiacovo F, Busceti CL, Biagioni F, Moyanova SG, Meisler MH, Battaglia G, Caricasole A, Bruno V, Nicoletti F (2009) Induction of the Wnt antagonist, Dickkopf-1, contributes to the development of neuronal death in models of brain focal ischemia. J Cereb Blood Flow Metab 29:264-276.

Mudher A, Chapman S, Richardson J, Asuni A, Gibb G, Pollard C, Killick R, Iqbal T, Raymond L, Varndell I, Sheppard P, Makoff A, Gower E, Soden PE, Lewis P, Murphy M, Golde TE, Rupniak HT, Anderton BH, Lovestone S (2001) Dishevelled regulates the metabolism of amyloid precursor protein via protein kinase $\mathrm{C} /$ mitogen-activated protein kinase and c-Jun terminal kinase. J Neurosci 21:4987-4995.

Niehrs C (2006) Function and biological roles of the Dickkopf family of Wnt modulators. Oncogene 25:7469-7481.

Rosi MC, Luccarini I, Grossi C, Fiorentini A, Spillantini MG, Prisco A, Scali C, Gianfriddo M, Caricasole A, Terstappen GC, Casamenti F (2010) Increased Dickkopf-1 expression in transgenic mouse models of neurodegenerative disease. J Neurochem 112:1539-1551.

Scali C, Caraci F, Gianfriddo M, Diodato E, Roncarati R, Pollio G, Gaviraghi G, Copani A, Nicoletti F, Terstappen GC, Caricasole A (2006) Inhibition of Wnt signaling, modulation of Tau phosphorylation and induction of neuronal cell death by DKK1. Neurobiol Dis 24:254-265.

Selkoe DJ (2002) Alzheimer's disease is a synaptic failure. Science 298:789-791

Shankar GM, Bloodgood BL, Townsend M, Walsh DM, Selkoe DJ, Sabatini BL (2007) Natural oligomers of the Alzheimer amyloid-beta protein induce reversible synapse loss by modulating an NMDA-type glutamate receptor-dependent signaling pathway. J Neurosci 27:2866-2875.

Svitkina TM, Verkhovsky AB, Borisy GG (1995) Improved procedures for electron microscopic visualization of the cytoskeleton of cultured cells. J Struct Biol 115:290-303.

Terry RD, Masliah E, Salmon DP, Butters N, DeTeresa R, Hill R, Hansen LA, Katzman R (1991) Physical basis of cognitive alterations in Alzheimer's disease: synapse loss is the major correlate of cognitive impairment. Ann Neurol 30:572-580.

Walsh DM, Selkoe DJ (2004) Deciphering the molecular basis of memory failure in Alzheimer's disease. Neuron 44:181-193.

Yaccoby S, Ling W, Zhan F, Walker R, Barlogie B, Shaughnessy JD Jr (2007) Antibody-based inhibition of DKK1 suppresses tumor-induced bone resorption and multiple myeloma growth in vivo. Blood 109:2106-2111. 